\title{
Availability of Antimalarial Drugs and Evaluation of the Attitude and Practices for the Treatment of Uncomplicated Malaria in Bangui, Central African Republic
}

\author{
Alexandre Manirakiza, ${ }^{1}$ Siméon Pierre Njuimo, ${ }^{1}$ Alain Le Faou, ${ }^{1}$ Denis Malvy, \\ and Pascal Millet ${ }^{2}$ \\ ${ }^{1}$ Institut Pasteur de Bangui, Avenue Pasteur, BP 923, Central African Republic \\ ${ }^{2}$ Université Victor Segalen Bordeaux 2, 146 rue Léo Saignat, 33076 Bordeaux Cedex, EA3677, France \\ Correspondence should be addressed to Alexandre Manirakiza, amanirak@yahoo.fr \\ Received 8 September 2009; Accepted 22 January 2010 \\ Academic Editor: Sukla Biswas
}

Copyright (C) 2010 Alexandre Manirakiza et al. This is an open access article distributed under the Creative Commons Attribution License, which permits unrestricted use, distribution, and reproduction in any medium, provided the original work is properly cited.

\begin{abstract}
National malaria management policy is based upon the availability of effective and affordable antimalarial drugs. This study was undertaken to evaluate the quality of the treatment of uncomplicated malaria cases in Bangui, an area with multidrug-resistant parasites, at a time preceding implementation of a new therapeutic policy relying on the artemisinin derivative combined treatment artemether-lumefantrine. A cross-sectional study was carried out in Bangui city to assess availability of antimalarial drugs and the performances of health workers in the management of uncomplicated malaria. Availability of drugs was recorded in all drugs wholesalers $(n=3)$, all pharmacies in health facilities $(n=14)$, private drugstores $(n=15)$, and in 60 non-official drug shops randomly chosen in the city. Despite a limited efficacy at the time of the survey, chloroquine remained widely available in the official and nonofficial markets. Artemisinin derivatives used in monotherapy or in combination were commonly sold. In health care facilities, $93 \%$ of the uncomplicated malaria cases were treated in the absence of any laboratory confirmation and the officially recommended treatment, amodiaquine-sulfadoxine/pyrimethamine, was seldom prescribed. Thus, the national guidelines for the treatment of uncomplicated malaria are not followed by health professionals in Bangui. Its use should be implemented while a control of importation of drug has to be reinforced.
\end{abstract}

\section{Background}

The aim of malaria management policy is to provide therapeutic guidelines to health workers and efficacious and affordable drugs to health facilities for patients to buy. This policy must be based on proper laboratory diagnosis and treatment of malarial episodes.

Given the rapid evolution of parasite resistance to chloroquine and sulfadoxine-pyrimethamine (SP), several countries have modified their national policy and adopted the artemisinin derivative combined treatments (ACT's) as recommended by WHO [1].

Access to drugs is widely available for the urban population through private drugstores and an illicit market [2]. The consequence is an increase of "drugs pressure" on the parasite strains, thus increasing the risk of development of multidrug resistance [3]. Moreover, urban malaria has its own epidemiological characteristics requiring appropriate fighting methods [4].

In Bangui, capital of Central African Republic (CAR), chloroquine is no longer recommended by the National Malaria Control Program (NMCP) because the resistance rate was of $40.9 \%$ in 2004 [5], while, in rural areas, this rate has been estimated to be around 15\% [6]. As a matter of fact, the artemether-lumefantrin (AL) combination should be implemented within a year from the period when this study is conducted. Thus, in the meantime, the amodiaquine and sulfadoxine-pyrimethamine (AQ/SP) association has been adopted as the first line treatment since year 2003. 
TABLE 1

\begin{tabular}{lll}
\hline Sample population: drugs sellers categories & Data collection method & Sample size \\
\hline $\begin{array}{l}\text { Generic drugs wholesalers (UCM, } \\
\text { CENTRAPHARM and ROFFE-PHARMA) }\end{array}$ & $\begin{array}{l}\text { Interview/questionnaire/assessment } \\
\text { of availability }\end{array}$ & 3 (exhaustive sample) \\
Health facilities & $\begin{array}{l}\text { Interview/questionnaire/assessment } \\
\text { of availability }\end{array}$ & 14 (exhaustive sample) \\
Private pharmacies ("Drugstores") & $\begin{array}{l}\text { Interview/questionnaire/assessment } \\
\text { of availability }\end{array}$ & 15 (exhaustive sample) \\
Drug vendors on the illicit market (city shops) & $\begin{array}{l}\text { Assessment of availability } \\
\text { (investigator behaved as a client) }\end{array}$ & 60 shops randomly chosen across the city \\
\hline
\end{tabular}

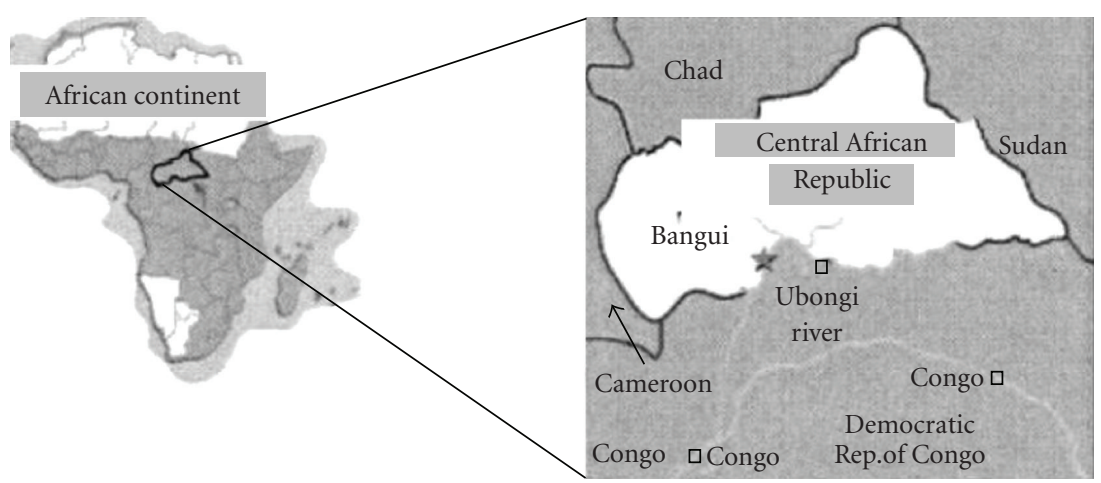

Figure 1: Location of Bangui, Central African Republic.

This study, conducted in 2006, reports the availability of antimalarial drugs in health facilities, drugstores, and illicit market, as well as the attitude of health workers for the diagnostic and treatment of uncomplicated malaria cases in health facilities in Bangui.

\section{Methods}

2.1. Study Area and Setting. This survey was carried out between May and July 2006 in Bangui, the capital of Central African Republic. The location of this city is shown in Figure 1.

This city is located on the Oubangui River which separates CAR from the Democratic Republic of Congo $\left(4^{\circ}\right.$ North, $19^{\circ}$ East). The climate is subequatorial with a rainfall period between April and November and temperatures varying from $19^{\circ} \mathrm{C}$ to $32^{\circ} \mathrm{C}$. This city of 620,771 inhabitants is subdivided in eight districts and represents $16.0 \%$ of the CAR population. Health care is provided by 14 small public health facilities, 4 private dispensaries, and 3 public hospitals. According to the NMCP guidelines, the management of an uncomplicated malarial episode is as follows: (i) clinical presumption of malaria, (ii) confirmation by microscopic diagnosis on blood film, and (iii) administration of the recommended first line treatment (currently AQ/SP). Quinine remains the first line antimalarial drug for pregnant women and the drug of choice for complicated malaria cases.

Malaria transmission is perennial, with a peak during the rainy season and is hyperendemic. In order to control the drug distribution chain, importation of all health products is submitted to a registration by the Ministry of Health and Population. As reported by the National Office of Medicines, two main categories of wholesalers are allowed to import generic drugs: a public centre ("Unité de Cession du Médicament," UCM) and two private wholesalers (CENTRAPHARM and ROFFE-PHARMA). UCM has been created following the Initiative of BAMAKO in 1987. It imports affordable generic drugs which are distributed to the health facilities. Additionally 17 private pharmacies import also drugs directly from various international wholesalers.

\subsection{Sampling Methods and Data Collection. Our method of} survey of the drugs providers is detailed in Table 1.

In the city shops, from the illicit market, interviewer asked for the availability of antimalarial drugs. The investigator had acted as a client and no questionnaire was filled up.

Assessment of uncomplicated malaria treatment has been achieved at all of the fourteen public health facilities and three private dispensaries of Bangui, where a questionnaire was used to collect data.

For all patients who had had a diagnosis of uncomplicated malaria and exit, an interview and a checking of their health notebook was done to know the way of obtaining diagnosis (laboratory confirmed or presumptive diagnosis) and which treatment has been prescribed. At the end of the day, health workers who were in charge of malaria patients were interviewed using a qualitative questionnaire. The theoretical knowledge and practical attitudes towards the content of the NMCP guidelines were collected. 
In order to minimize the Hawthorne effect (the Hawthorne effect is defined as a "positive and temporary change in some measurable behavior in a situation where the observer had no intention to truly affect the other individual's behavior") [7] in malaria management practices, in each health facility the assessment was only performed in one day.

2.3. Data Analysis. Data were entered and analyzed on EpiInfo software 3.3.2, (Centers for Disease Control and Prevention, Atlanta, USA). The results were presented as proportions.

2.4. Ethical Approval. The complete study protocol was accepted, duly registered, and approved by the CAR Ministry of Health and Population, Malaria division. An oral informed consent was given by all interviewed participants at the official drug market and health facilities. For the purpose of this study, the nonsofficial drug sellers were kept blind of the investigator intention, as they would have been reluctant to disclose information because their activity is considered as illegal in CAR.

\section{Results}

3.1. Antimalarial Drugs Availability in Bangui. In Bangui, the type of antimalarial drug availability depends widely upon the category of provider (Table 2).

Amodiaquine, sulfadoxine-pyrimethamine, artemether, halofantrine, and quinine were available from the three generic drugs wholesalers. Two ACT's combinations: artesunate-sulfamethoxypyrazine-pyrimethamine (Coarinate) and artemether-lumefantrine (Coartem) were available in two private wholesalers' stores.

SP, and Q were available from all "official" drug providers. Drugstores sold the complete panel of antimalarial drugs including chloroquine which was absent from wholesalers and health centers. CQ was also widely present in the illegal market, while AQ, SP and Q are sometimes available. Drugstores were the only place where Art-C were present. However it has to be noted that AT was widely available on the official market despite the recommendation to not use it alone.

3.2. Therapeutic Practice for Uncomplicated Malaria. Among 42 patients treated for uncomplicated malaria, only three (7.1\%) were prescribed antimalarial drug based upon microscopy results. Recommended AQ/SP was prescribed only in $14.3 \%$ of cases (Figure 2 ).

A total of 18 health workers out of $25(72 \%)$ confirmed that they prescribed treatment in the absence of laboratory diagnosis arguing that fever is strongly associated with malaria in an endemic area, and such an attitude allows to exclude for the patient thick and thin blood film cost which is officially charged 0.76 USD.

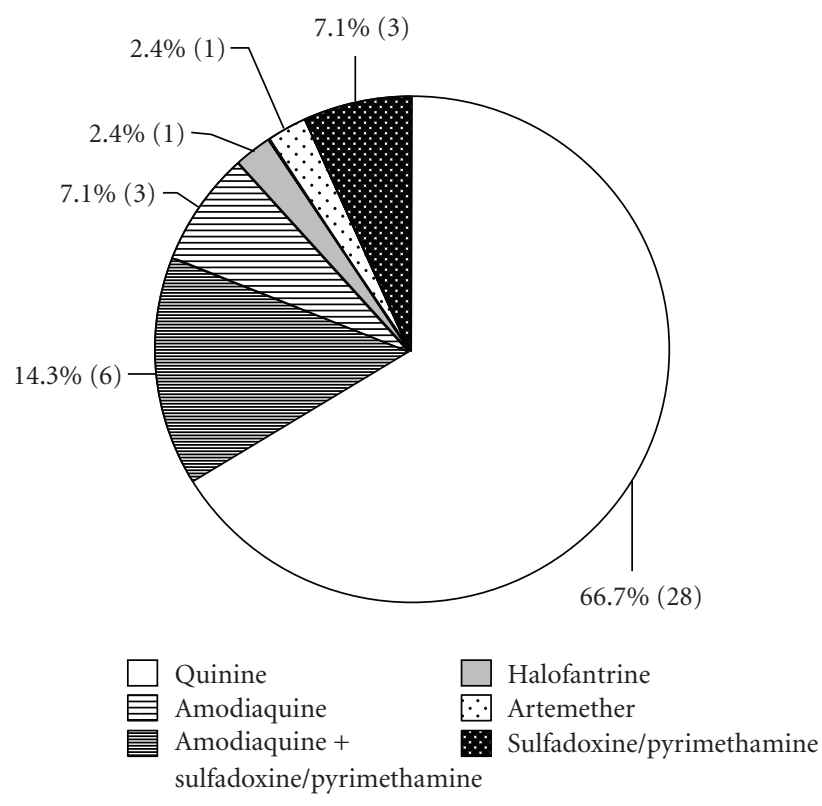

Figure 2: Types of prescription of antimalarial drugs in Bangui health facilities, May-July 2006 assessment.

A basic knowledge of the official guidelines was reported for 10 out of 23 health workers (44\%) regarding children, 13 out of $20(65 \%)$ regarding pregnant women and 14 out of 25 (56\%) regarding other categories of patients.

\section{Discussion}

The survey has shown that the management of malaria is not satisfactory because a wide range of antimalarial drugs remains available in Bangui city, distributed among the official and illicit market, and the poor quality of service offered in health centres.

Although chloroquine is no longer officially recommended and has been withdrawn from the public health facilities, it remains available in drugstores and city shops. Despite a loss of efficacy $[5,6]$, it is still widely used because it is the cheapest antimalarial drug available, and, because it was given the status of intermediate resistance in CAR, since it is active against malaria symptoms without curing parasitemia.

The illicit drug market is generalized in developing countries $[8,9]$ as trafficking across the borders is poorly controlled. The price of drugs found in such market is attractive (these drugs are often product of poor quality and of a dubious origin) and they are retailed as tablets for 1 single treatment $[10,11]$. In India, Das and Hammer noted that the private market encourages inadequate treatments whatever can be the competence of the providers [12].

In 2006, WHO asked for the withdrawal of artemisinin as monotherapy, used in Bangui, as it may be responsible for a rapid emergence of resistance [1] which would impair its future use in combination therapies. However, it remains available in Bangui where it is commonly used as a therapeutic option. 
TABLE 2: Number of providers of antimalarial drugs in Bangui, May to July 2006 assessment.

\begin{tabular}{|c|c|c|c|c|c|c|c|}
\hline Provider (Total) & AQ & AT & CQ & Art-C & $\mathrm{HA}$ & SP & Q \\
\hline Generic drugs at wholesalers $(n=3)$ & 3 & 3 & - & 2 & 3 & 3 & 3 \\
\hline Generic drugs sold at pharmacies at health facilities $(n=14)$ & 14 & 3 & - & - & - & 14 & 14 \\
\hline Drugstores $(n=15)$ & 11 & 13 & 14 & 14 & 11 & 15 & 15 \\
\hline Non official drugs shops or illicit market $(n=60)$ & 11 & - & 42 & - & - & 10 & 11 \\
\hline
\end{tabular}

AQ: Amodiaquine, AT: Artemether, CQ: Chloroquine, Art-C: Artemether Combinations, HA: Halofantrine, SP: Sulfadoxine-Pyrimethamine, Q: Quinine.

Many factors hamper the changing of therapeutic habits and henceforth the adoption of international recommendations. Changing the national malaria treatment policy is challenging and costly. In Tanzania, for instance, replacing chloroquine with SP would require 4 percent of the annual government budget [13]. The diversity of antimalarial drugs available is also an obstacle to the success of the national malaria treatment policy [14]. Thus the number of available drugs should be limited and people should be informed of the reason for such a policy.

Attendance in health facilities was very low at the time of the study (42 cases of uncomplicated malaria diagnosed). According to health workers, defection of the community for health facilities is due to the costs of consultation and treatment as they consider that fever is always associated with malaria. Thus self-medication or drugstore counseling is favoured.

The absence of microscopic confirmation is a common practice among health workers in Bangui as well as in others developing countries. This attitude is of concern as there is no algorithm for malaria treatment based solely on clinical examination [15]. As reported in other countries [16], systematic treatment is given to minimize the cost for patients with limited income. However, according to a study in Malawi, which compared systematic treatment to microscopic confirmation, the latter attitude would allow annual savings of 17,472 USD per medical facility [17].

Legal and universal medical practice implementation requires validated specific training before allowing health workers to consult and prescribe drugs. Nevertheless, in developing countries like CAR, due to lack of qualified practitioners, paramedics are receiving in-service training and are then considered capable of taking medical decisions. It have been reported that theoretical skill alone has little impact on practical behaviour when dealing with malaria patients [18], and that financial incentives to practitioners do not lead to an improvement in quality of health whereas ongoing training organized by health authorities has a significant one [12].

\section{Conclusion}

Our study indicates that the treatment of malaria was poorly handled in the health facilities of Bangui in 2006. The availability of antimalarials on the illegal market without any assurance about their quality and the fact that health workers do not follow the official guidelines are obstacles to the success of the government's policy for the control of malaria. Chloroquine should be withdrawn from CAR drug market.

Training, information, and participation of health professionals to decision making are a key to improve adherence to new protocol guidelines. Results from this study and other investigations conducted in other countries highlight the need to consider specific implementation guidelines since most African countries have now adopted ACT's combination therapies. Considering that artemether-lumefantrine and artesunate-amodiaquine have been made available for first line treatment since the beginning of 2007 in CAR (NMCP 2008 reports), ongoing evaluations of the compliance of health workers to the new guidelines are indispensable as well as the acceptation of these drugs by the population.

\section{Acknowledgments}

Sponsorship: a grant for this study was provided by Ministère française des Affaires étrangères et de la Coopération. The authors thank the Central African Malaria Program Group: Nestor Madji, Pierrette Kokessa, and Roger Max Koula for their technical support.

\section{References}

[1] WHO, "Guidelines for the treatment of malaria," WHO/HTM/MAL/2006.1108, June 2006, http://whqlibdoc .who.int/publications/2006/9241546948_eng_full.pdf.

[2] A. Niandou, Marché parallèle des médicaments au Niger: exemple de la communauté urbaine de Niamey, Thése de doctorat en pharmacie, Dakar, Senegal, 1993.

[3] P. Olliaro, "Drug resistance hampers our capacity to roll back malaria," Clinical Infectious Diseases, vol. 41, supplement 4, pp. S247-S257, 2005.

[4] V. Robert, K. Macintyre, J. Keating, et al., "Malaria transmission in urban sub-Saharan Africa," American Journal of Tropical Medicine and Hygiene, vol. 68, no. 2, pp. 169-176, 2003.

[5] D. Menard, N. Madji, A. Manirakiza, D. Djalle, M. R. Koula, and A. Talarmin, "Efficacy of chloroquine, amodiaquine, sulfadoxine-pyrimethamine, chloroquine-sulfadoxine-pyrimethamine combination, and amodiaquine-sulfadoxine- pyrimethamine combination in Central African children with noncomplicated malaria," American Journal of Tropical Medicine and Hygiene, vol. 72, no. 5, pp. 581-585, 2005.

[6] I. Bergeri, D. D. Zoguéreh, N. Madji, et al., "In vivo evaluation of chloroquine therapeutic efficacy in uncomplicated Plasmodium falciparum malaria in Central African Republic 
in 1997 and 1998," Bulletin de la Societe de Pathologie Exotique, vol. 96, no. 1, pp. 29-34, 2003.

[7] K. Leonard and M. C. Masatu, "Outpatient process quality evaluation and the Hawthorne effect," Social Science and Medicine, vol. 63, no. 9, pp. 2330-2340, 2006.

[8] D. Fassin, "La vente illicite des médicaments au Sénégalet et ses Conséquences pour la santé des populations," Bulletin of the Exotic Pathology Society, vol. 79, pp. 557-570, 1996.

[9] J. M. Trapsida, "Mieux cerner le marché illicite des médicaments en Afrique, pour une lutte plus efficace," Communication pour la table ronde de ReMeD, Octobre 1999.

[10] O. M. S. Minzi, M. J. Moshi, D. Hipolite, et al., "Evaluation of the quality of amodiaquine and sulphadoxine/pyrimethamine tablets sold by private wholesale pharmacies in Dar Es Salaam Tanzania," Journal of Clinical Pharmacy and Therapeutics, vol. 28, no. 2, pp. 117-122, 2003.

[11] A. A. Amin, R. W. Snow, and G. O. Kokwaro, "The quality of sulphadoxine-pyrimethamine and amodiaquine products in the Kenyan retail sector," Journal of Clinical Pharmacy and Therapeutics, vol. 30, no. 6, pp. 559-565, 2005.

[12] J. Das and J. S. Hammer, Strained Mercy: The Quality of Medical Care in Delhi, Policy Research Working Paper Series, no. 3228, The World Bank, The Institute of Socio-Economic Research on Development and Democracy, Washington, DC, USA, 2004, http://ssrn.com/abstract=610269.

[13] J.-A. Mulligan, R. Mandike, N. Palmer, et al., "The costs of changing national policy: lessons from malaria treatment policy guidelines in Tanzania," Tropical Medicine and International Health, vol. 11, no. 4, pp. 452-461, 2006.

[14] H. A. Williams, D. Durrheim, and R. Shretta, "The process of changing national malaria treatment policy: lessons from country-level studies," Health Policy and Planning, vol. 19, no. 6, pp. 356-370, 2004.

[15] D. Chandramohan, S. Jaffar, and B. Greenwood, "Use of clinical algorithms for diagnosing malaria," Tropical Medicine and International Health, vol. 7, no. 1, pp. 45-52, 2002.

[16] D. Zurovac, B. A. Larson, W. Akhwale, and R. W. Snow, "The financial and clinical implications of adult malaria diagnosis using microscopy in Kenya," Tropical Medicine and International Health, vol. 11, no. 8, pp. 1185-1194, 2006.

[17] A. Jonkman, R. A. Chibwe, C. O. Khoromana, et al., "Costsaving through microscopy-based versus presumptive diagnosis of malaria in adult outpatients in Malawi," Bulletin of the World Health Organization, vol. 73, no. 2, pp. 223-227, 1995.

[18] D. Zurovac, A. K. Rowe, S. A. Ochola, et al., "Predictors of the quality of health worker treatment practices for uncomplicated malaria at government health facilities in Kenya," International Journal of Epidemiology, vol. 33, no. 5, pp. 10801091, 2004. 


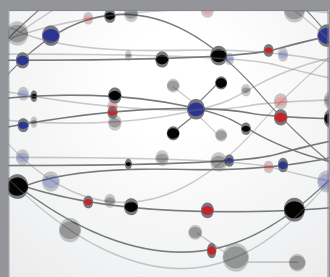

The Scientific World Journal
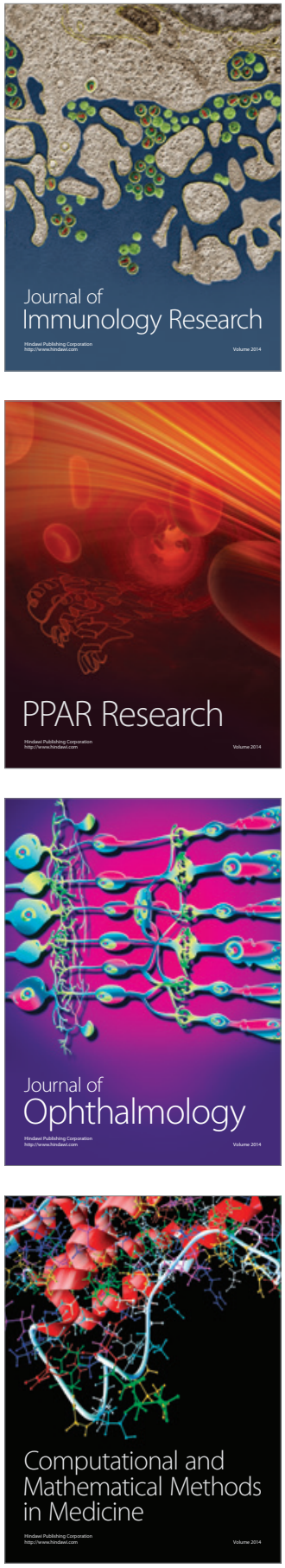

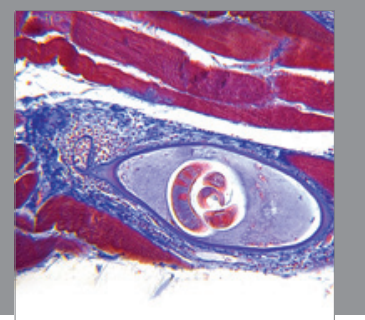

Gastroenterology

Research and Practice
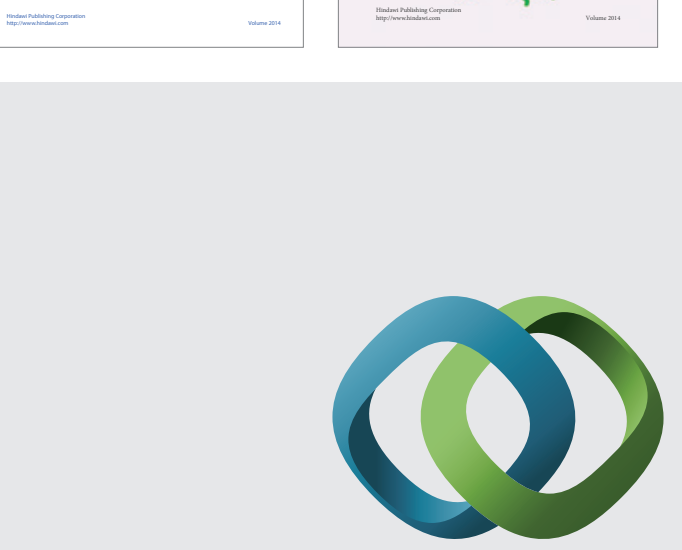

\section{Hindawi}

Submit your manuscripts at

http://www.hindawi.com
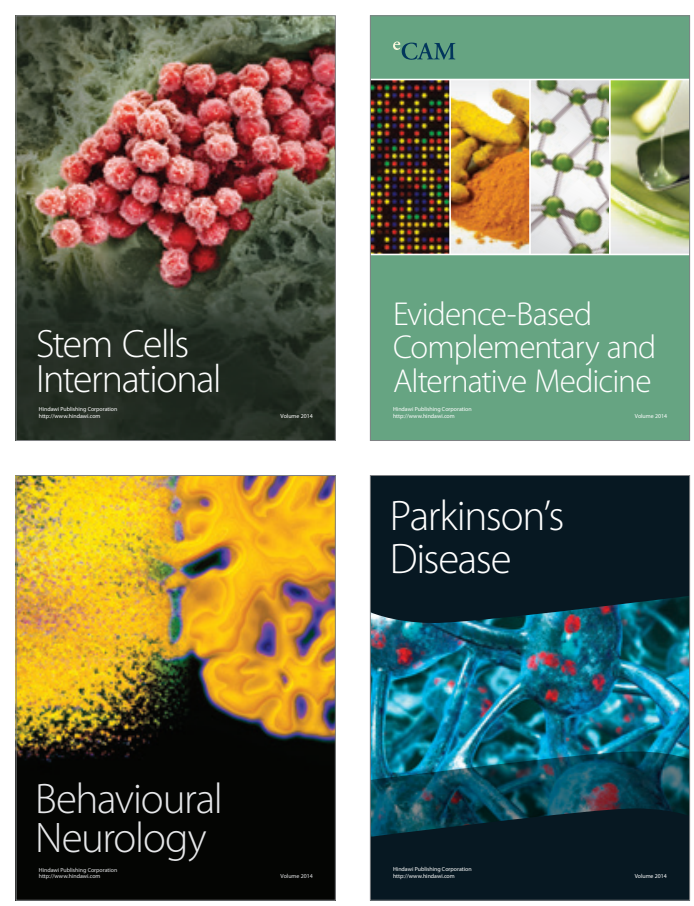

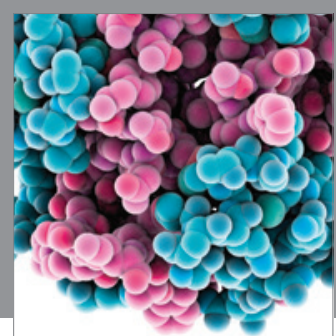

Journal of
Diabetes Research

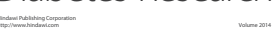

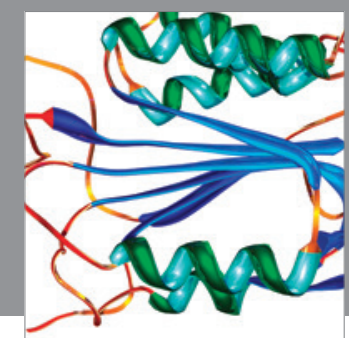

Disease Markers
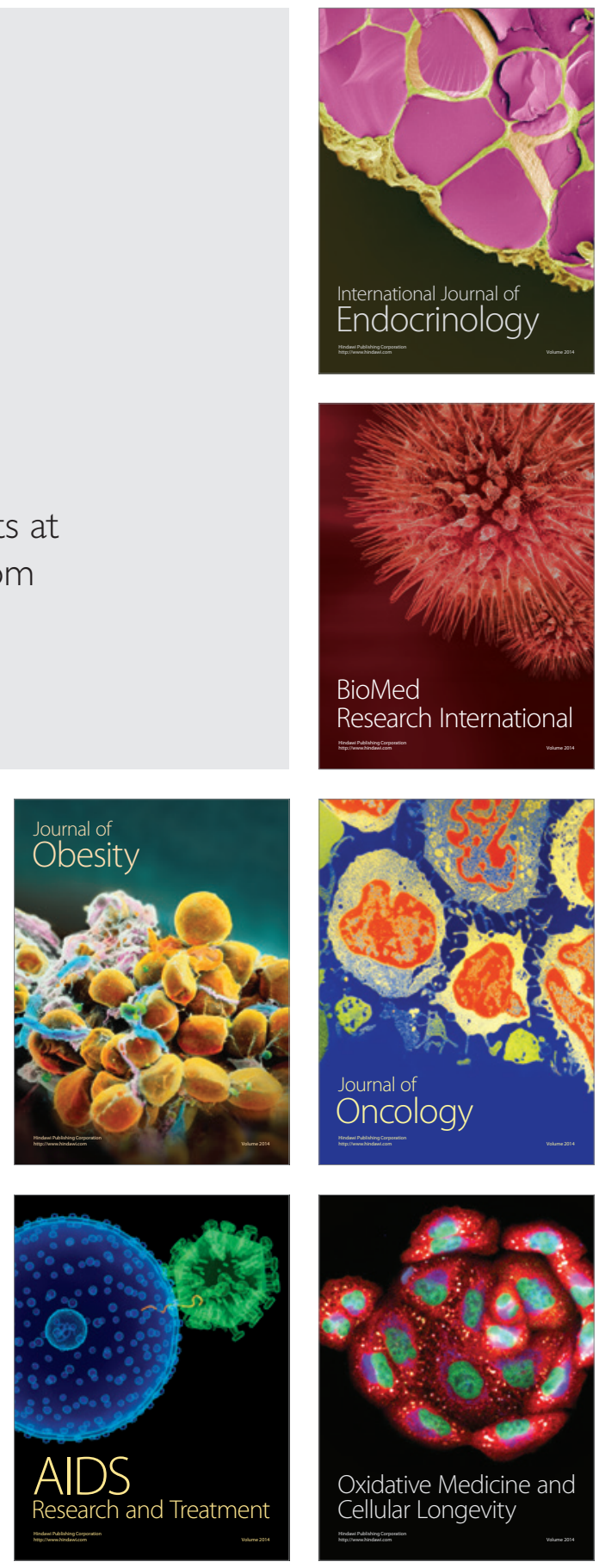